\title{
Digger wasps of the families Ampulicidae and Sphecidae (Hymenoptera: Apoidea) in the collection of the Institute of Systematics and Ecology of Animals, Novosibirsk, Russia
}

\section{Роющие осы семейств Ampulicidae и Sphecidae (Hymenoptera: Apoidea) в комлекщии Института систематики и экологии жиивотных СО РАН, Новосибирск}

\author{
Yu.N. Danilov \\ Ю.Н. Ааницов
}

Institute of Systematics and Ecology of Animals, Russian Academy of Sciences, Siberian Branch, Frunze Str. 11, Novosibirsk 630091 Russia. E-mail: prionyx@mail.ru

Институт систематики и экологии животных СО РАН, ул. Фрунзе 11, Новосибирск 630091 Россия

Key words: digger wasps, Hymenoptera, Catalog, Museum, Collection.

Ключевые слова: роющие осы, перепончатокрылые, каталог, музей, коллекция.

Abstract. 2996 specimens (1636 males and 1360 females) of the families Ampulicidae and Sphecidae belonging to 119 species from 16 genera and 6 subfamilies were examined. Types of four sphecid wasp species are deposited in the collection of Siberian Zoological Museum of Institute of Systematics and Ecology of Animals, Novosibirsk, Russia.

Резюме. Изучено 2996 экземпляров $\left(16360^{7} 0^{7}\right.$ и 1360 우) роющих ос семейств Ampulicidae и Sphecidae из 119 видов, 16 родов и 6 подсемейств. Типовые серии четырёх видов сфецид хранятся в коллекции Сибирского зоологического музея Института систематики и экологии животных СО РАН.

\section{Introduction}

The Museum was founded in 1960 as a laboratory named Zoological Museum in the Biological Institute of the Siberian Division of the Academy of Science of the USSR (currently the Institute of Systematics and Ecology of Animals, Russian Academy of Sciences, Siberian Branch).

Abbreviation of the Museum is SZMN (ISEN or ISEA follow the name of Institute in different publications).

The fund collection of digger wasps began to be actively replenished with material in the 1990s, mainly due to the collections of V.V. Dubatolov. Also, a significant contribution to the development of the collection made by A.V. Barkalov, V.K. Zintshenko, O.E. Kosterin (Novosibirsk), A.S. Shlyakhtenok (Minsk, Belarus).

Since 2007, the collection has also been replenished with materials by Yu.N. Danilov, A.M. Byvaltsev (Novosibirsk).

Proshchalykin M.Yu., V.M. Loktionov and A.S. Lelej (Vladivostok), M.V. Mokrousov (Nizhny Novgorod) and D.A. Milko (Bishkek, Kyrgyzstan) have made a significant contribution to the collection in recent years. Some material was kindly provided to the collection by T. Ljubomirov (Sofia, Bulgaria).

A number of publications were prepared based on the collection material: Danilov, 2008; 2009; 2011a, b; 2012a, b; 2013; 2014a, b; 2015; 2017 a, b, c; Danilov, Tshernyshev, 2008; Danilov, Odintseva, 2013; Danilov, Mokrousov, 2017a, b; Danilov, Byvaltsev, 2020.

The classification of sphecid wasps is based on Pulawski [2020]. Subfamilies and genera are listed alphabetically.

\section{Ampulicidae}

Ampulicinae

Ampulex Jurine, 1807

Ampulexcompressa (Fabricius, 1781)

Material. 1우 from Thailand.

\section{Dolichurinae}

Dolichurus Latreille, 1809

Dolichurus bicolor

Lepeletier de Saint Fargeau, 1845

Material. $2 \sigma^{7} \sigma^{\urcorner}$from Russia (Crimea Rep.).

Dolichurus corniculus (Spinola, 1807)

Material. 19 from Belarus.

Dolichurus turanicus Gussakovskij, 1952

Material. $10^{7}$ from Tajikistan.

\section{Sphecidae \\ Ammophilinae}

Ammophila W. Kirby, 1798

Ammophila adelpha Kohl, 1901

Material. 4 우 from Kazakhstan.

Ammophila asiatica Tsuneki, 1971

Material. $60^{7} 0^{7}$, 290 from Russia (Tuva Rep.) and Kazakhstan. 


\section{Ammophila barkalovi Danilov, 2015}

Type material. $10^{7}, 2 \circ \circ$. Holotype: $\circ$, Kazakhstan: Almaty region, Altyn-Emel National park, near mount Ayak-Kalkhan, 26.IV.2012, A.V. Barkalov leg. Paratypes: 10, Kazakhstan: Almaty region, Altyn-Emel National park, near mount AyakKalkhan, 23.IV.2012, V.K. Zintshenko leg. 1우 Kazakhstan: Almaty region, Altyn-Emel National park, near mount AyakKalkhan, 1-4.V.2012, A.V. Barkalov, Yu.N. Danilov leg.

Ammophila beaumonti Dollfuss, 2013

Material. 19 from Mongolia.

Ammophila beniniensis

(Palisot de Beauvois, 1806)

Material. $10^{7}$ from South Africa.

Ammophila campestris Latreille, 1809

Material. 390 $0^{7} 0^{7}$, 28우 from Russia (Volgograd Prov., Omsk Prov., Novosibirsk Prov., Altai Terr., Altai Rep., Khakass Rep., Tuva Rep.) and Kazakhstan.

Ammophila clavus (Fabricius, 1775)

Material. $70^{7} 0^{7}$, 8우 from Vietnam and Thailand.

Ammophila deserticola Tsuneki, 1971

Material. $810^{7} \sigma^{7}$, 28우 from Russia (Kurgan Prov., Kemerovo Prov., Altai Terr., Altai Rep., Khakass Rep., Tuva Rep., Irkutsk Prov., Zabaikalskii Terr., Amur Prov.) and Mongolia.

Ammophila elongata Fischer de Waldheim, 1843

Material. $30^{\top} \sigma^{\top}, 2 \circ \circ$ from Russia (Volgograd Prov.) and Kazakhstan.

Ammophila formosensis Tsuneki, 1971

Material. $230^{7} \sigma^{7}$, 4 ㅇ from Tajikistan and Kyrgyzstan.

Ammophila gracillima Taschenberg, 1896

Material. $2 \bigcirc^{\top} \sigma^{\top}, 1$ from Russia (Astrakhan Prov.) and Turkmenistan.

Ammophila haladai Dollfuss, 2013

Material. $40^{7} \sigma^{7}$, $69+$ from Russia (Omsk Prov., Novosibirsk Prov., Khakass Rep.), Kazakhstan and Mongolia.

\section{Ammophila heydeni Dahlbom, 1845}

Material. 1220 $0^{7} 0^{7}$, 63ㅇ from Russia (Astrakhan Prov., Dagestan Rep., Omsk Prov., Novosibirsk Prov., Altai Terr.), Armenia, Turkmenistan, Kyrgyzstan, Tajikistan and Kazakhstan.

Ammophila hungarica Mocsáry, 1883

Material. $150^{7} 0^{7}$, 15 우 from Russia (Dagestan Rep., Omsk Prov., Novosibirsk Prov., Altai Terr.), Turkmenistan, Uzbekistan, Kyrgyzstan and Tajikistan.

Ammophila infesta F. Smith, 1873

Material. $60^{7} \sigma^{7}$, 4 ㅇ from Russia (Primorskii Terr.).

$$
\begin{gathered}
\text { Ammophila kondarensis } \\
\text { Danilov, } 2018
\end{gathered}
$$

Material. $10^{x}, 1$ 우 from Uzbekistan and Tajikistan.

Ammophila laevigata F. Smith, 1856

Material. 2o+ from Thailand.
Ammophila mongolensis Tsuneki, 1971

Material. $70^{7} \sigma^{7}$, 1 ㅇ from Russia (Khakass Rep., Buryat Rep., Zabaikalskii Terr.), Kyrgyzstan and Kazakhstan.

Ammophila occipitalis F. Morawitz, 1890

Material. $20^{7} 0^{7}$ from Turkmenistan.

Ammophila producticollis Morice, 1900

Material. $10^{7}$ from Turkmenistan.

Ammophila pubescens Curtis, 1836

Material. $770^{7} 0^{7}$, 79 우 from Russia (Kurgan Prov., Tyumen Prov., Tomsk Prov., Novosibirsk Prov., Altai Terr., Altai Rep., Khakass Rep., Tuva Rep., Irkutsk Prov., Buryat Rep., Zabaikalskii Terr., Primorskii Terr.) and Belarus.

Ammophila rauschi Dollfuss, 2013

Material. $40^{7} 0^{7}$, 5 우우 from Kyrgyzstan and Tajikistan.

Ammophila rufipes Guérin-Méneville, 1831

Material. 1 우 from Chile.

\section{Ammophila sabulosa (Linnaeus, 1758)}

Material. 1680 $0^{7} 0^{7}$, 127우 from Russia (Voronezh Prov., Saratov Prov., Rostov Prov., Astrakhan Prov., KabardinoBalkarian Rep., Dagestan Rep., Kurgan Prov., Tyumen Prov., Omsk Prov., Tomsk Prov., Novosibirsk Prov., Altai Terr., Altai Rep., Khakass Rep., Tuva Rep., Krasnoyarsk Terr., Irkutsk Prov., Yakutsk Rep., Zabaikalskii Terr., Kamchatka Terr.), Finland, Belarus, Kyrgyzstan and Kazakhstan.

Ammophila sarekandana Balthasar, 1957

Material. $200^{7} \sigma^{7}$, 6우 from Tajikistan. Ammophila sareptana Kohl, 1884

Material. $20^{7} \sigma^{7}, 1+$ from Kazakhstan.

Ammophila separanda F. Morawitz, 1891

Material. $20^{7} \sigma^{7}$, 2 우 from Russia (Astrakhan Prov., Dagestan Rep.).

Ammophila sickmanni Kohl, 1901

Material. 1 f from Russia (Zabaikalskii Terr.). Ammophila sinensis Sickmann, 1894

Material. $90^{7} \sigma^{7}$, 14우 from Russia (Omsk Prov., Novosibirsk Prov., Altai Terr., Khakass Rep., Tuva Rep., Buryat Rep.).

Ammophila striata Mocsáry, 1879

Material. $1+$ from Kyrgyzstan.

Ammophila tekkensis Gussakovskij, 1930

Material. 10 $10^{7}, 1$ from Kazakhstan.

Ammophila terminata terminata $\mathrm{F}$. Smith, 1856

Material. 440 $0^{7}$, 26우 from Russia (Volgograd Prov., Astrakhan Prov., Omsk Prov., Novosibirsk Prov., Altai Terr., Altai Rep., Khakass Rep., Tuva Rep.), Hungary, Kyrgyzstan, Kazakhstan and Mongolia.

Ammophila terminata turanica Kazenas, 1998

Material. $20^{\top} \sigma^{\top}, 1+$ from Kazakhstan.

Ammophila terminata turkestana Kohl, 1906

Material. 10', 390 from Kazakhstan. 
Ammophila tsunekii Menke, 1976

Material. 107, 1 우 from Mongolia.

Ammophila vagabunda F. Smith, 1856

Material. 200 $\sigma^{7}$, 20우 from Russia (Amur Prov., Khabarovsk Terr., Primorskii Terr.) and Japan (Honshu).

Ammophila vetuberosa Q. Li et J. Yang, 1994

Material. $10^{7}, 19$ from Mongolia.

Eremnophila Menke, 1964

Eremnophila aureonotata (Cameron, 1888)

Material. $4 \bigcirc^{7} \sigma^{7}$, 3우 from USA and Canada.

Eremochares Gribodo, 1883

Eremochares dives (Brullé, 1833)

Material. $110^{7} 0^{7}$, 7 우 from Russia (Kalmykia Rep., Astrakhan Prov., Dagestan Rep.), Turkmenistan, Kyrgyzstan and Kazakhstan.

Hoplammophila de Beaumont, 1960

Hoplammophila aemulans (Kohl, 1901)

Material. $60^{\top} \sigma^{7}$, $69+$ from Russia (Primorskii Terr.).

Hoplammophila clypeata (Mocsáry, 1883)

Material. $10^{\top}, 1$ 우 from Bulgaria.

Parapsammophila Taschenberg, 1869

Parapsammophila caspica (Gussakovskij, 1930)

Material. 3우 from Turkmenistan.

Parapsammophila turanica F. Morawitz, 1890

Material. 1 우 from Turkmenistan

Podalonia Fernald, 1927

Podalonia affinis (W. Kirby, 1798)

Material. 1100' $0^{7}, 102$ 오 from Russia (Omsk Prov., Novosibirsk Prov., Altai Terr., Altai Rep., Khakass Rep., Tuva Rep., Zabaikalskii Terr., Amur Prov., Khabarovsk Terr.), Tajikistan, Kyrgyzstan, Kazakhstan, Mongolia and China.

Podalonia albohirsuta (Tsuneki, 1971)

Material. 1 우 from Kazakhstan.

\section{Podalonia alpina (Kohl, 1888)}

Material. $110^{7} 0^{7}$, 4 웅 from Russia (Altai Rep., Tuva Rep.) Kyrgyzstan and Tajikistan.

Podalonia asiatica Danilov, 2017

Type Material. $\left(30^{7} \sigma^{7}\right)$. Holotype: $\sigma^{7}$, Russia: Tuva Republic: Tes-Khemsky District, near Ak-Erik, 8-9.VI.2010, Yu. Danilov leg. Paratypes: $10^{7}$, Russia: Tuva Republic: Tes-Khemsky District, near Despen, 25.VI.1948. 107, Russia: Tuva Republic: BayTayginsky District, river Eldig-Khem, 8.VI.2008, R. Dudko, I. Lyubechansky leg.

Material. $90^{\top} 0^{\top}$ from Russia (Altai Rep., Tuva Rep.) and Tajikistan.

Podalonia atrocyanea (Eversmann, 1849)

Material. $70^{7} 0^{7}$, 11우 from Russia (Khakass Rep., Tuva Rep., Zabaikalskii Terr.) and Mongolia.

Podalonia caucasica (Mocsáry, 1883)

Material. 420 $0^{7} 0^{7}$, 70우 from Russia (Omsk Prov., Novosibirsk Prov., Altai Terr., Altai Rep., Tuva Rep., Irkutsk
Prov., Zabaikalskii Terr.), Tajikistan, Kyrgyzstan, Kazakhstan and Mongolia.

Podalonia chalybea (Kohl, 1906)

Material. $40^{\top} 0^{\top}, 2+9$ from Mongolia.

Podalonia ebenina (Spinola, 1839)

Material. $60^{7} 0^{7}, 13+9$ from Turkmenistan, Tajikistan and Kazakhstan.

Podalonia fera (Lepeletier de Saint Fargeau, 1845)

Material. 140 $0^{7}$, 14우 from Russia (Volgograd Prov., Kalmykia Rep., Astrakhan Prov., Dagestan Rep.), Turkey, Tajikistan and Kazakhstan.

\section{Podalonia flavida (Kohl, 1901)}

Material. 900 $0^{7}$, 4990 from Russia (Volgograd Prov., Omsk Prov., Novosibirsk Prov., Altai Terr., Khakass Rep., Tuva Rep., Buryat Rep., Zabaikalskii Terr.), Tajikistan, Kazakhstan and Mongolia.

Podalonia gobiensis (Tsuneki, 1971)

Material. $180^{7} \sigma^{7}$, 24우우 from Russia (Tuva Rep.).

Podalonia gussakovskii Danilov, 2017

Material. 1 우 from Tajikistan.

Podalonia hirsuta (Scopoli, 1763)

Material. 106 $0^{7} 0^{7}$, 119우 from Russia (Dagestan Rep., Crimea Rep., Tyumen Prov., Novosibirsk Prov., Kemerovo Prov., Altai Terr., Altai Rep., Tuva Rep., Buryat Rep., Zabaikalskii Terr.), Belarus, Turkmenistan, Tajikistan, Kyrgyzstan and Kazakhstan.

Podalonia hirsutaffinis (Tsuneki, 1971)

Material. $20^{7} \sigma^{7}$ from Kyrgyzstan.

Podalonia luffii (E. Saunders, 1903)

Material. 420 $0^{\top} \sigma^{7}$, 23우 from Russia (Voronezh Prov., Astrakhan Prov., Altai Terr., Tuva Rep.), Kyrgyzstan and Kazakhstan.

Podalonia nazarovae Danilov, 2017

Type material. $\left(15 \sigma^{\top} \odot^{7}, 2 \circ\right)$ : Holotype: $\odot^{7}$, Tajikistan: Tavildara District, Kishlak Dehi-Colon near Tavildara, 1025.V.2016, Yu.N. Danilov, A.V. Barkalov, V.K. Zinchenko leg. Paratypes: $140^{\top} 0^{7}, 2$ 우, same data as holotype.

Material. $50^{7} \sigma^{7}, 2$ 우 from Tajikistan.

Podalonia nigrohirta (Kohl, 1888)

Material. $20^{\top} \sigma^{\top}, 13$ 웅 from Uzbekistan, Tajikistan and Kazakhstan.

Podalonia pungens (Kohl, 1901)

Material. $20^{7} 0^{7}, 209$ from Kazakhstan.

Podalonia tydei (Le Guillou, 1841)

Material. $80^{7} 0^{7}$, 11우 from Russia (Astrakhan Prov.) Turkey, Kyrgyzstan, Tajikistan and Kazakhstan.

Chloriontinae

Chlorion Latreille, 1802

Chlorion lobatum (Fabricius, 1775)

Material. 2 2 9 from Thailand.

Chlorion magnificum F. Morawitz, 1887

Material. $2 \circ 9$ from Turkmenistan and Thailand. 
Chlorion maxillosum (Poiret, 1787)

Material. 1 우 from South Africa.

Chlorion regale $\mathrm{F}$. Smith, 1873

Material. $90^{7} \sigma^{7}, 1+$ from Turkmenistan.

Sceliphrinae

Sceliphrini

Chalybion Dahlbom, 1843

Chalybion bengalense (Dahlbom, 1775)

Material. $140^{7} \sigma^{7}$, 9우 from Yemen, Thailand, Vietnam and Malaysia.

Chalybion californicum (de Saussure, 1763)

Material. $30^{7} 0^{7}, 1$ 우 from USA and Canada.

Chalybion femoratum (Fabricius, 1781)

Material. 2 우 from Tajikistan and Kyrgyzstan.

Chalybion japonicum (Gribodo, 1883)

Material. 10 $10^{7}$, 우 from Russia (Amur Prov.), China (Guangdong) and Japan (Honshu).

Chalybion madecassum (Gribodo, 1883)

Material. 3 우 from Seychelles.

Chalybion omissum (Kohl, 1889)

Material. 1 iq from Georgia.

Chalybion turanicum (Gussakovskij, 1935)

Material. $50^{7} 0^{7}$, 11우 from Tajikistan, Kyrgyzstan and Kazakhstan.

Chalybion walteri (Kohl, 1889)

Material. 10 $10^{7}$, 4 우 from Iran and Turkmenistan.

Sceliphron Klug, 1801

Sceliphron caementarium (Drury, 1773)

Material. $30^{7} \sigma^{7}, 13$ 우 from Japan (Honshu), USA and Canada.

Sceliphron coromandelicum

(Lepeletier de Saint Fargeau, 1845)

Material. 1 우 from Thailand.

Sceliphron curvatum (F. Smith, 1870)

Material. 10 $10^{x}$, 59 from Russia (Voronezh Prov., Krasnodar Terr., Crimea Rep.) and Bulgaria.

Sceliphron deforme (F. Smith, 1856)

Material. $50^{7} \sigma^{7}$, 24 우 from Russia (Novosibirsk Prov., Krasnoyarsk Terr., Amur Prov., Khabarovsk Terr., Primorskii Terr.), Tajikistan and Kyrgyzstan.

Sceliphron destillatorium (Illiger, 1807)

Material. 110 $0^{7} \sigma^{7}$, 24우 from Russia (Astrakhan Prov., Crimea Rep., Altai Terr.), Turkmenistan, Tajikistan, Kyrgyzstan and Kazakhstan.

Sceliphron fuscum Klug, 1801

Material. $20^{7} \sigma^{7}$, 5 우 from Seychelles.

Sceliphron madraspatanum (Fabricius, 1781)

Material. 190 $0^{7}$, 25 +9 from Russia (Astrakhan Prov., Krasnodar Terr.), Uzbekistan, Tajikistan, Croatia, China (Guangdong), Japan (Ryukyu), Vietnam and Thailand.
Sceliphron shestakovi Gussakovskij, 1928

Material. 5우 from Tajikistan and Turkmenistan.

Sceliphron spirifex (Linnaeus, 1758)

Material. 10, 3 우 from Turkey, Yemen and Rwanda.

Sphecinae

Prionychini

Chilosphex Menke, 1976

Chilosphex argyrius (Brullé, 1833)

Material. $100^{7} O^{7}$, 5 우 from Russia (Dagestan Rep.) and Croatia.

Palmodes Kohl, 1890

Palmodes hissaricus Danilov, 2020

Material. $\left(50^{7} 0^{7}, 290\right)$ : Paratypes: $10^{7}, 19$, Iran: Mazandaran Province: Elburs Mts., 60 km W Damghan, vic. Foulad Mahalleh, 2000-2300 m, 4.VII.2010, A. Timokhov leg. 10², Kazakhstan: Karagandy Province: near Balkhash city, 10.VI.2009, Yu. Danilov leg. 1으, Kazakhstan: Karagandy Province: Zhanaarka District, Schalginskij, 4-8.VI.1937, Chievina leg. $20^{7} 0^{7}$, Kyrgyzstan: Talass Province: Manas District, near Pokrovka, 03.VI.2005, D. Milko leg. $10^{7}$, Tajikistan: Gorno-Badakhshan Province: near Khorugh, 14.VII.2018, V. Zintshenko leg.

Palmodes mandarinius (F. Smith, 1856)

Material. $70^{7} \sigma^{7}$, 8 우 from Russia (Altai Rep., Tuva Rep., Zabaikalskii Terr.), Mongolia and Tajikistan.

Palmodes melanarius (Mocsáry, 1883)

Material. 160 $160^{7}$, 5 우 from Russia (Dagestan Rep., Kalmykia Rep., Astrakhan Prov.), Tajikistan, Kyrgyzstan and Kazakhstan.

Palmodes minor (F. Morawitz, 1890)

Material. $30^{7} 0^{7}$, 6우우 from Tajikistan, Kyrgyzstan, Kazakhstan and Iran.

Palmodes occitanicus

(Lepeletier de Saint Fargeau

et Audinet-Serville, 1828)

Material. 210 $0^{\top} \sigma^{\top}$, 9 웅 from Russia (Dagestan Rep., Altai Terr., Tuva Rep.), Tajikistan, Kyrgyzstan, Kazakhstan and Iran.

Palmodes orientalis (Mocsáry, 1883)

Material. $80^{\top} 0^{\top}$, 5 우 from Russia (Orenburg Prov., Tuva Rep., Buryat Rep., Zabaikalskii Terr.), Kazakhstan.

Palmodes strigulosus (A. Costa, 1861)

Material. 400 $\sigma^{7}$, 14우우 from Russia (Krasnodar Terr., Dagestan Rep.), Turkmenistan, Uzbekistan, Kyrgyzstan and Tajikistan.

Prionyx Vander Linden, 1827

Prionyx (Calosphex) haberhaueri

(Radoszkowski, 1871)

Material. $10^{\top}$ from Kyrgyzstan.

Prionyx (Calosphex) nigropectinatus (Taschenberg, 1869)

Material. 10 $10^{7}$ 우 from Kazakhstan.

Prionyx (Calosphex) niveatus (Dufour, 1854)

Material. $40^{7} \mathrm{O}^{7}$, 18 우 from Russia (Astrakhan Prov.), Tajikistan and Kazakhstan. 


\section{Prionyx (Harpactopus) subfuscatus (Dahlbom, 1845)}

Material. $310^{7} \sigma^{7}$, 14 우 from Russia (Kalmykia Rep., Astrakhan Prov., Krasnodar Terr., Altai Terr., Tuva Rep., Buryat Rep., Primorskii Terr.), Kyrgyzstan, Tajikistan and Kazakhstan.

Prionyx (Neosphex) spinolae (F. Smith, 1856)

Material. 1 우 from Chile.

\section{Prionyx (Prionyx) kirbii \\ (Vander Linden, 1827)}

Material. 220 $0^{7} 0^{7}$, 39우 from Russia (Astrakhan Prov., Dagestan Rep., Altai Terr.), Kyrgyzstan and Kazakhstan.

Prionyx (Prionyx) lividocinctus (A. Costa, 1861)

Material. $50^{7} \sigma^{7}$, 590 from Russia (Kalmykia Rep., Astrakhan Prov., Dagestan Rep., Crimea Rep.) and Kyrgyzstan.

Prionyx (Prionyx) nudatus (Kohl, 1885)

Material. 220 $\sigma^{7} \sigma^{7}$, 21우 from Russia (Astrakhan Prov., Dagestan Rep., Omsk Prov., Novosibirsk Prov., Altai Terr., Tuva Rep.), Kyrgyzstan and Kazakhstan.

Prionyx (Prionyx) songaricus (Eversmann, 1849)

Material. $10^{\top}, 1$ 우 from Turkmenistan.

Prionyx (Prionyx) viduatus viduatus (Christ, 1791)

Material. 160 $0^{7}$, 16우 from Russia (Kalmykia Rep., Astrakhan Prov.), Tajikistan, Kyrgyzstan, Kazakhstan and Japan (Ryukyu)

Prionyx (Prionyx) viduatus mocsaryi (Kohl, 1885)

Material. 210 $\sigma^{7} \sigma^{7}$, 12 우 from Russia (Volgograd Prov., Omsk Prov., Altai Terr., Tuva Rep.) and Kazakhstan.

\section{Sphecini}

Isodontia Patton, 1880

Isodontia mexicana (de Saussure, 1867)

Material. 3 우 from Canada.

Isodontia nigella (F. Smith, 1856)

Material. $20^{7} \sigma^{7}$, 3 우 from Japan (Honshu, Ryukyu).

Isodontia paludosa (Rossi, 1790)

Material. 19 from Turkey.

Sphex Linnaeus, 1758

Sphex argentatus Fabricius, 1787

Material. $20^{7} \sigma^{7}, 4+9$ from Japan (Kyushu, Ryukyu) and Thailand.

Sphex atropilosus Kohl, 1885

Material. $40^{7} \sigma^{7}$, 1 우 from Russia (Volgograd Prov., Astrakhan Prov., Novosibirsk Prov.).

Sphex diabolicus F. Smith, 1858

Material. $40^{7} \sigma^{7}$, 690 from Japan (Ryukyu) and Vietnam.

Sphex flavipennis Fabricius, 1793

Material. $150^{7} 0^{x}$, 9 우 from Russia (Dagestan Rep.), Tajikistan, Kyrgyzstan and Kazakhstan.

\section{Sphex fumicatus Christ, 1791}

Material. $10^{7}, 1$ 우 from Turkey.
Sphex funerarius Gussakovskij, 1934

Material. $640^{7} \sigma^{7}$, 38ㅇ from Russia (Astrakhan Prov., Dagestan Rep., Orenburg Prov., Kurgan Prov., Omsk Prov., Novosibirsk Prov., Altai Terr., Altai Rep., Tuva Rep., Buryat Rep., Zabaikalskii Terr.), Ukraine, Kyrgyzstan, Tajikistan, Kazakhstan and Mongolia.

Sphex ichneumoneus (Linnaeus, 1758)

Material. 2 우 from Canada.

Sphex leuconotus Brullé, 1833

Material. 120 $0^{7} 0^{7}$, 8 우 from Russia (Astrakhan Prov., Dagestan Rep.), Kyrgyzstan and Kazakhstan.

Sphex melas Gussakovskij, 1930

Material. $10^{\top}, 1$ 우 from Turkmenistan.

Sphex oxianus Gussakovskij, 1928

Material. $40^{7} \sigma^{7}$, 5 우 from Turkmenistan, Tajikistan and Kyrgyzstan.

Sphex pensylvanicus Linnaeus, 1763

Material. $10^{\top}$ from Canada.

Sphexpruinosus Germar, 1817

Material. $120^{7} \sigma^{7}$, 19 from Russia (Astrakhan Prov., Dagestan Rep.), Croatia and Turkey.

Sphex sericeus (Fabricius, 1804)

Material. $10^{7}, 1+$ from Thailand.

\section{Conclusion}

The family Ampulicidae is very poorly represented in the collection - 4 species ( $2 \%$ of the World fauna).

The family Sphecidae is represented by 115 species, which is $14.5 \%$ of the World fauna. The vast majority of species in the collection are Palaearctic - 95 species ( $35 \%$ of the Palaearctic fauna). Materials from Russia belong to 52 species, which is $75 \%$ of the country's fauna.

\section{Acknowledgements}

I am very grateful to Arkady S. Lelej (Vladivostok, Russia) who reviewed the manuscript and made important suggestions.

The study was supported by The Federal Fundamental Scientific Research Programme for 2013-2020 (AAAA-A16116121410121-7).

\section{References}

Danilov Yu.N. 2008. The digger wasps (Hymenoptera: Sphecidae, Crabronidae) of the Altai forest-steppe near Barnaul// Euroasian Entomological Journal. Vol.7. No.4. P.345-352. [In Russian]

Danilov Yu.N. 2009. The fauna of digger wasps (Hymenoptera: Sphecidae) of the Kulundinskaya Steppe and adjacent territories // A.I. Kurentsov's Annual Memorial Meetings. Vol.20. P.52-62. [In Russian].

Danilov Yu.N. 2011a. Digger wasps of the family Sphecidae (Hymenoptera: Apoidea) of Daurskii Reserve // A.I. Kurentsov's Annual Memorial Meetings. Vol.22. P.199206. [In Russian]. 
Danilov Yu.N. 2011b. New records of digger wasps of the family Sphecidae (Hymenoptera, Apoidea) from the Asian part of Russia // Euroasian Entomological Journal. Vol.10. No.2. P.188-190. [In Russian].

Danilov Yu.N. 2012a. To the knowledge of the digger wasps of the tribe Prionychini Bohart and Menke, 1963 (Hymenoptera, Sphecidae) of Russia and adjacent countries // Proceedings of the Russian Entomological Society. Vol.83. No.1. P.159165. [In Russian].

Danilov Yu.N. 2012b. The digger wasps of the genus Prionyx Vander Linden (Hymenoptera: Sphecidae) of Russia and Central Asia, with a key to species, new synonymies, and lectotype designations // Zootaxa. Vol.3526. P.59-71.

Danilov Yu.N. 2013. New records of the digger wasp Palmodes orientalis (Mocsáry) (Apoidea, Sphecidae) in Eurasia // Euroasian Entomological Journal. Vol.12. No.1. P.46-50. [In Russian].

Danilov Yu.N. 2014a. Review of Sphecidae wasps (Hymenoptera: Apoidea) of Siberia. Part I. List of species // Euroasian Entomological Journal. Vol.13. No.5. P.422-429. [In Russian].

Danilov Yu.N. 2014b. Review of Sphecidae wasps (Hymenoptera: Apoidea) of Siberia. Part 2. Key to genera and species // Euroasian Entomological Journal. Vol.13. No.6. P.511521. [In Russian].

Danilov Yu.N. 2015. New and little known digger wasps of the genus Ammophila W. Kirby, 1798 (Hymenoptera: Apoidea: Sphecidae) from Central Asia // Zootaxa. Vol.3964. P.537545.

Danilov Yu.N. 2017a. Taxonomic notes on Palearctic Podalonia Fernald, 1927 (Hymenoptera, Sphecidae), with descriptions of four new species // Zootaxa. Vol.4320. No.3. P.554-570.

Danilov Yu.N. 2017b. Family Ampulicidae - Ampulicid digger wasps // Belokobylskij S.A., Lelej A.S. (Eds). Annotated catalogue of the Hymenoptera of Russia. Volume I. Symphyta and Apocrita: Aculeata. Proceedings of the Zoological Institute of the Russian Academy of Sciences. Supplement 6. P.211

Danilov Yu.N. 2017c. Family Sphecidae - Sphecid digger wasps // in S.A. Belokobylskij and A.S. Lelej (editors). Annotated catalogue of the Hymenoptera of Russia. Volume I. Symphyta and Apocrita: Aculeata. Proceedings of the Zoological Institute of the Russian Academy of Sciences. Supplement 6. P.212-216.

Danilov Yu.N., Byvaltsev A.M. 2020. The digger wasps of the genus Palmodes Kohl, 1890 in Central Asia (Hymenoptera: Sphecidae: Prionychini) // Zootaxa. Vol.4803. No.3. P.401434.

Danilov Yu.N., Mokrousov M.V. 2017a. New data on distribution and taxonomy of some Palaearctic species of Sphecidae (Hymenoptera: Apoidea) // Euroasian Entomological Journal. Vol.16. No.2. P.107-113.

Danilov Yu.N., Mokrousov M.V. 2017b. New records of sphecid digger wasps (Hymenoptera: Apoidea: Sphecidae) in Russia // Proceedings of the Russian Entomological Society. Vol.88. No.2. P.53-55.

Danilov Yu.N., Odintseva A.A. 2013. To our knowledge of digger wasp fauna (Hymenoptera: Sphecidae, Crabronidae) of south taiga of Irtysch River, West Siberia, Russia // Euroasian Entomological Journal. Vol.12. No.4. P.331-335. [In Russian].

Danilov Yu.N., Tshernyshev S.E. 2008. Digger wasps (Hymenoptera: Sphecidae, Crabronidae) in the Kulundinskaya forest-steppe of West Siberia // Euroasian Entomological Journal. Vol.7. No.1 P.40-46. [In Russian].

Pulawski W.J. 2020. Catalog of Sphecidae. California Academy of Sciences, San Francisco. Available from: http:// researcharchive.calacademy.org/research/entomology/ entomology resources/hymenoptera/sphecidae/ family_group_names_and_classification.pdf. 